\title{
Variación Estacional del Peso vivo en Machos Caprino Criollo, en el Sur de Mendoza
}

\author{
Dayenoff, $P^{1 y 3} ;$ Dri, $P^{2} ;$ Macario, J. ${ }^{3}$; Poblete, $R^{4}$ \\ ${ }^{1}$ Facultad de Cs. Veterinarias y Ambientales. Universidad Juan Agustín Maza. Guaymallén. \\ Mendoza \\ ${ }^{2}$ Dirección Provincial de Ganadería de Mendoza. Rivadavia 867. 5600 San Rafael. Mendoza. \\ ${ }^{3}$ INTA EEA Rama Caída. El vivero s/n. 5603 Rama Caída. Mendoza. \\ ${ }^{4}$ Producto privado. Ojo de Agua. Malargüe, Mendoza. \\ Correo electrónico: pdayenoff@yahoo.com.ar
}

\section{RESUMEN}

El objetivo del presente trabajo fue evaluar la variación del peso vivo de machos caprinos Criollo, estudiados dentro del Plan Castrón, al inicio y al final del período de servicio, en la zona sur de Mendoza. Para ello se utilizaron 824 machos tipo Criollo, divididos según edad por cronología dentaria. El peso vivo se evaluó con una balanza electrónica de $150 \mathrm{~kg}$ de capacidad máxima, con $50 \mathrm{~g}$ de precisión, en el mes de mayo (otoño austral), al inicio de la etapa reproductiva y en septiembre (primavera austral), al momento que los chivos fueron retirados de los establecimientos productores por "el castronero". El diseño experimental respondió a un modelo completamente aleatorizado, evaluando las medias y desvíos estándar entre los diferentes momentos del trabajo por test de Tukey aplicando el programa estadístico InfoStat 2.0. Los resultados mostraron pesos de 28,13 $\pm 4,4$ y $27,94 \pm 5.1 \mathrm{~kg}$ para los machitos de 2 dientes, en otoño y septiembre, respectivamente; $52,35 \pm 4,7$ y $41,92 \pm 7,4 \mathrm{~kg}$ para los machos de 4 dientes, $68,4 \pm 6,3$ y $56,7 \pm 6,7 \mathrm{~kg}$ para los chivos de 6 dientes, 78,84 $\pm 6,2$ y $62,3 \pm 7,6 \mathrm{~kg}$ para los machos de boca llena y $70,4 \pm 6,9$ y $57,4 \pm 7,3 \mathrm{~kg}$ para los chivos de medio diente, existiendo una diferencia estadística significativa $(p \leq 0,05)$ entre los dos períodos evaluados para los animales de 4 y 6 dientes, boca llena y medio diente. Se concluye que el peso vivo de los chivos Criollo seleccionados en Plan Castrón muestran una variación estacional significativa entre el inicio de la estación reproductiva (otoño austral) y el final de la etapa de servicio 
(primavera austral) influenciada por la condición del pastizal natural para cada momento y la actividad de los machos durante la estación reproductiva.

Palabras clave: caprinos Criollo, machos, peso vivo, variación estacional.

\section{Seasonal variation of live weight in Criollo bucks, in Southern Mendoza}

\section{ABSTRACT}

The objective of the present study was to evaluate the variation of the live weight of Creole goats males studied within the Castron Plan at the beginning and at the end of the service period, in the South of Mendoza. For that purpose, 824 Creole type males, divided according to age by dental chronology, were used. Live weight was evaluated with an electronic scale of $150 \mathrm{~kg}$ maximum capacity, with $50 \mathrm{~g}$ of precision, in May (austral autumn), at the beginning of the reproductive stage and in September (austral spring), at the time that the goats were removed from the producing establishments by "the castronero". The experimental design responded to a completely randomized model, evaluating standard means and deviations between the different work moments by Tukey test applying the statistical program InfoStat 2.0. The results showed 28.13 weights \pm 4.4 and $27.94 \pm 5.1 \mathrm{~kg}$ for males with 2 teeth, in September, and autumn respectively; $52.35 \pm 4.7$ and $41,92 \pm 7,4 \mathrm{~kg}$ for males with 4 teeth, males $68.4 \pm 6.3$ and $56.7 \pm 6,7$ $\mathrm{kg}$ for the goats with 6 teeth, 78,84 \pm 6.2 and $62,3 \pm 7,6 \mathrm{~kg}$ for males with full mouth and $70.4 \pm 6,9$ and $57.4 \pm 7,3 \mathrm{~kg}$ for the goats with half tooth, there is a significant statistical difference $(p \leq 0.05)$ between the two periods evaluated for animals 4-6 teeth, mouth full and half tooth. We conclude showing the live weight of the selected Creole goats in Plan Castron a significant seasonal variation between the onset of the reproductive season (austral autumn) and the end of the service stage (austral spring) influenced by the condition of the natural grassland for every moment and the males' activity during the reproductive season.

Key words: Criollo goats, male, live weight, seasonal variation.

Fecha de recepción artículo original: 02-05-2018

Fecha de aceptación para su publicación: 10-11-2018 


\section{Introducción}

El peso vivo de un macho caprino se puede definir como el incremento de peso y el tamaño corporal en una unidad de tiempo concreta, influenciado por la raza, la alimentación, la época de nacimiento, el manejo y las enfermedades que sufrió el animal, entre otras causas ${ }^{(1)}$.

Asimismo, Gbangboche, et al (2006) ${ }^{(2)}$ mencionaron la importancia del año de nacimiento como factor de influencia sobre la evolución de peso de machos a distintas edades y Mabrouk, et al (2010) ${ }^{(3)}$ encontraron una variación importante en el peso de los chivos, en diferentes edades y épocas del año, aún dentro de la misma agrupación racial.

Este cambio significativo de peso se observa sobre todo después del año de edad cuando la evolución de ese parámetro comienza a aplanarse. ${ }^{(3)}$

Un efecto similar describieron Ndlovu y Simela $(1996)^{(4)}$ en chivos de Zimbabwe, situación que estaría vinculada a la variación estacional de lluvias y a la producción de forraje en las regiones áridas y semi-áridas donde se desarrolla principalmente la producción caprina para carne ${ }^{(5)}$, reportada, a su vez por Dadi, et al $(2008)^{(6)}$ y por Zhang, et al $(2009)^{(7)}$, autores que vincularon la variación estacional del peso de los chivos a distintas edades, con factores netamente climáticos.

En Australia, Mc Gregor $(2010)^{(8)}$ halló para machos Angora las mismas características descriptas y, según el autor, se solucionaría a través de una suplementación estratégica en la época seca.

Asimismo, Gaddour et al., $2007^{(9)}$ resalta que esa variación estacional del peso del chivo se inicia en el período de lactancia, momento en que el cabrito depende exclusivamente del estado de alimentación de la madre, mientras que Deribe y Taye $(2013)^{(10)}$ encontraron que dependiendo de la estación del año y de la producción forrajera del mismo, los chivitos pueden crecer $65 \mathrm{~g}$ /día en la estación seca y hasta 160 g/día en la estación húmeda.

Por otra parte, Jimmy, et al $(2010)^{(11)}$ reportan sobre la importancia del manejo que se realiza en el establecimiento sobre el peso adulto de los chivos, sobre todo en los cuidados de una alimentación eficiente y un manejo sanitario correcto, como también lo describen Chenyambuga y Lekule (2014)(12).

Cabe destacar que la región del sur de Mendoza responde a una zona árida fría, con precipitaciones estivo-otoñales que alcanzan los $200 \mathrm{~mm} / \mathrm{año}$ y que muestra influencia directa sobre la productividad forrajera de la región ${ }^{(13)}$ logrando su mejor calidad alimenticia en el mes de enero. ${ }^{(14)}$ 
El objetivo del presente trabajo fue evaluar la variación estacional del peso vivo en chivos Criollo de la región de Malargüe, teniendo en cuenta la variable calidad forrajera de los arbustos consumidos en esas estaciones.

\section{Material y método}

El presente trabajo se llevó a cabo dentro de las actividades del Plan Castrón que realizan desde hace 4 años distintas instituciones vinculadas a la producción caprina de Mendoza, en los Departamentos de Malargüe y San Rafael, donde hay una población caprina de aproximadamente 650 mil cabezas, con un promedio de 480 caprinos por establecimiento, existiendo en la región aproximadamente 2.200 ganaderías mixtas con predominancia de ganado caprino.

El clima de la región de tipo árido, templado-frío, con veranos de temperatura que llegan a los $39^{\circ} \mathrm{C}$ e invierno muy fríos con temperaturas de hasta $-14^{\circ} \mathrm{C}$ y nevadas intensas que llegan a los 3 metros de nieve en la cordillera media y alta y precipitaciones que varían de este a oeste entre los 240 y $120 \mathrm{~mm}$ por año, concentradas en el período primavera-estival.

La vegetación es de tipo monte bajo achaparrado predominando el estrato arbustivo, con escasa presencia de gramíneas y ausencia de ejemplares del estrato arbóreo; siendo descripta como una zona de transición entre región del Monte y norte de Patagonia Argentina.

El manejo de los caprinos se realiza de forma empírica, con escasa aplicación de tecnología de base científica, los animales se alimentan bajo un sistema de pastoreo libre del pastizal natural, sin control de carga animal, no existe la suplementación estratégica, no se aplica un calendario sanitario preventivo mínimo, escaso nivel de mejoramiento genético racial e instalaciones inadecuadas a la cantidad de animales en cada establecimiento.

Este sistema de explotación caprina tiene dos características que la distinguen del resto de la producción caprina del país, "la veranada", sistema de trashumancia para el aprovechamiento de los pastizales de altura entre los meses de diciembre y abril y "el castronero", productor que se encarga de retirar los machos de los establecimiento entre los meses de septiembre y mayo, para concentrar los servicios en mayo-julio, evitando a las hembras entrar en servicio en verano y las pariciones de cabras en los meses de mayor frío (julio-agosto).

Para el presente trabajo se utilizaron 1364 machos caprinos tipo Criollo regional correspondiente a algo más de 220 ganaderías comerciales de la zona, descartando aquellos chivos que presentaron taras 
o defectos, los que son separados del grupo de Machos Seleccionados dentro del Plan Castrón

De los machos seleccionados, se evaluaron un total de 824 caprinos tipo Criollo regional en dos momentos del año, septiembre (primavera austral), a la salida de los animales de los establecimiento cuando son recogidos por "los castroneros" para llevarlos a "la veranada" al finalizar el período de reproducción, con un total de 443 animales y mayo (otoño austral), momento de regreso de los animales de "la veranada", al inicio de la época de servicio, evaluando 381 chivos.

Previo a la evaluación, los machos fueron identificados con caravanas numeradas en el pabellón auricular izquierdo; la evaluación del peso vivo se realizó por la mañana, utilizando una balanza electrónica de $150 \mathrm{~kg}$ de capacidad máxima y $50 \mathrm{~g}$ de precisión, colocando a los animales sobre un arnés colgado de un trípode de soporte.

Los valores se tomaron y asentaron diferenciando los chivos por edad, según cronología dentaria, machos dos dientes (D2), machos 4 dientes (D4), machos 6 dientes (D6), machos boca llena (D8) y machos medio diente (MD) y momento del año de la valoración.

El diseño experimental correspondió a un modelo completamente aleatorizado, realizándose el estudio de los datos según momento del año por medio de análisis varianza y test de Tukey con el paquete estadístico InfoStat 2.0.

\section{Resultados}

En la tabla 1 se ven los valores de los pesos (en $\mathrm{kg}$ ) de chivo Criollo $(\mathrm{n}=443)$ evaluados en el mes de septiembre, donde se aprecia que los chivitos D2 presentaron el menor peso promedio $(27,94 \pm 5,1 \mathrm{~kg})$ de los grupos evaluados en el período primaveral, destacándose que D6 y MD presentaron pesos similares $(56,7 \pm 6,7$ y $57,4 \pm 7,3 \mathrm{~kg})$ y observándose que D8 presentó el peso más elevado $(62,3 \pm 7,6 \mathrm{~kg})$ para ese momento de pesada.

Asimismo, la tabla muestra que para todos los rangos etarios evaluados el coeficiente de variación fue bajo, con un mínimo de 10,18 en los chivos D4 y un máximo de 13,14para los animales D2.

Además se observa que solo los rangos etarios D6 y MD no presentan diferencias estadística significativas $(p \leq 0,05)$ entre sí para el período de pesada de primavera. 
Tabla 1. Media, desviación estándar y coeficiente de variación (CV) del peso vivo de chivos Criollo, evaluados en el mes de Septiembre (primavera austral)

\begin{tabular}{|c|c|c|c|}
\hline Edad & $\mathbf{n}$ & Media \pm d.e. & CV \\
\hline D2 & 73 & $27,94 \pm 5,1^{\text {a }}$ & 13,4 \\
\hline D4 & 67 & $41,92 \pm 7,4^{\text {b }}$ & 10,18 \\
\hline D6 & 88 & $56,7 \pm 6,7^{\text {c }}$ & 11,2 \\
\hline D8 & 94 & $62,3 \pm 7,6^{\text {d }}$ & 12,72 \\
\hline MD & 59 & $57,4 \pm 7,3^{\text {c }}$ & 11,83 \\
\hline
\end{tabular}

Letras diferentes en la misma columna = diferencia estadística significativa $(\mathrm{p} \leq 0,05)$

Por otra parte, en la tabla se muestra una diferencia estadística significativa $(\mathrm{p} \leq 0,05)$ más destacada entre los chivos D2 con D4 y D6 y D8.

Tabla 2. Media, desviación estándar y coeficiente de variación (CV) del peso vivo de chivos Criollo, evaluados en el mes de Mayo (otoño austral)

\begin{tabular}{|c|c|c|c|}
\hline Edad & $\mathbf{n}$ & Media \pm d.e. & CV \\
\hline D2 & 87 & $28,13 \pm 4,4^{\text {a }}$ & 15,8 \\
\hline D4 & 90 & $52,35 \pm 4,7^{\text {b }}$ & 9,52 \\
\hline D6 & 92 & $68,4 \pm 6,3^{\text {c }}$ & 9,8 \\
\hline D8 & 101 & $78,84 \pm 6,2^{\text {d }}$ & 12,38 \\
\hline MD & 73 & $76,4 \pm 6,9^{\text {d }}$ & 11,47 \\
\hline
\end{tabular}

Letras diferentes en la misma columna = diferencia estadística significativa $(\mathrm{p} \leq 0,05)$

A su vez, la tabla muestra diferencias estadísticas significativas $(\mathrm{p} \leq 0,05)$ entre todas las edades salvo en el caso de los chivos de boca llena y medio diente, encontrando un bajo coeficiente de variación en todos las edades de los machos para este período.

En la Tabla 3 se observa la evolución del peso de los machos Criollo, en los dos momentos testeados, encontrando que para la misma edad, los chivos evaluados en Mayo mostraron pesos más elevados que los pesados en Septiembre (primavera austral), alcanzando su máximo peso a la edad de boca llena con $78,84 \pm 6,2 \mathrm{~kg}$ en la pesada del otoño austral. 
Tabla 3: Media y desviación estándar del peso vivo chivos Criollo, según momento de evaluación.

\begin{tabular}{|c|c|c|}
\hline Edad & $\begin{array}{c}\text { Septiembre (primavera } \\
\text { austral) }\end{array}$ & $\begin{array}{c}\text { Mayo } \\
\text { (otoño austral) }\end{array}$ \\
\hline D2 & $27,94 \pm 5,1^{\mathrm{a}}$ & $28,13 \pm 4,4^{\mathrm{a}}$ \\
\hline D4 & $41,92 \pm 7,4^{\mathrm{a}}$ & $52,35 \pm 4,7^{\mathrm{b}}$ \\
\hline D6 & $56,7 \pm 6,7^{\mathrm{a}}$ & $68,4 \pm 6,3^{\mathrm{b}}$ \\
\hline D8 & $62,3 \pm 7,6^{\mathrm{a}}$ & $78,84 \pm 6,2^{\mathrm{b}}$ \\
\hline MD & $57,4 \pm 7,3^{\mathrm{a}}$ & $70,4 \pm 6,9^{\mathrm{b}}$ \\
\hline
\end{tabular}

Letras diferentes en la misma fila $=$ diferencia estadística significativa $(p \leq 0,05)$

\section{Discusión}

La evolución de peso vivo de chivos Criollo según edad por dentición, evaluados en este trabajo, se comportó similar a lo mencionado por Rahman et al (2008) ${ }^{(15)}$ en chivos Black Bengal, con una variación de peso vinculada a la estacionalidad de lluvias y a la producción de forraje en las regiones áridas y semi-áridas, zonas donde se desarrolla principalmente la producción caprina de carne. ${ }^{(5)}$

Al igual que lo reportado por Dadi, et al $(2008)^{(6)}$ y Zhang, et al $(2009)^{(7)}$, los machos caprinos de Malargüe mostraron que la variación estacional del peso a distintas edades tiene una relación directa con factores netamente climáticos, mostrando que en el momento de mayor disponibilidad y calidad forraje ${ }^{(14)}$ presentan un mejor peso vivo como lo describieron Ndlovu y Simela $(1996)^{(4)}$ para los chivos de Zimbabwe.

Asimismo, los valores de peso hallados en los chivos Criollo son similares a los descriptos por Esteban, $2008^{(16)}$ y por Luque, 2011 $11^{(17)}$ para una de las razas españolas precursoras de la Criollo, como las razas Blanca Celtibérica, Retinta, Malagueña y a las razas Canarias, descriptas por Capote, et al (1998) ${ }^{(18)}$

A su vez, los chivos Criollo de Malargüe presentaron mayor peso adulto que el biotipo neuquino, de acuerdo a lo descrito por Lanari $(2004)^{(19)}$ y del biotipo formoseño encontrado por Revidetti et al $(2010)^{(20)}$ y al de los chivos locales de la región de Puebla ${ }^{(21)}$ como resultado den una alimentación eficiente y un manejo sanitario correcto, como también los describen Chenyambuga y Lekule (2014) ${ }^{(22)}$ 


\section{Conclusión}

Los chivos de raza Criollo de la región de Malargüe muestran una variación estacional de peso vivo relacionada con factores climáticos regionales que condicionan la calidad y disponibilidad forrajera y ese peso es superior al descrito para otros biotipos regionales de país. 


\section{Bibliografia}

1. Hamad, A. H. An evaluation of performance of crossbred dairy goats at Magadu Dairy farm. Dissertation for Award of MSc Degree at Sokoine University of Agriculture, Morogoro, Tanzania.2001; 136pp.

2. Gbangboche, A.B., Adamou-Ndiaye, M. and Youssao, A.K.I. Non-genetic factors affecting the reproduction performance, lamb growth and productivity indices of Djallonke sheep. Small Ruminant Research.2006; 64: 133-142.

3. Mabrouk. O., Sghaier, N., Costa, R.G., Amor,G., Ammar,B., Amel, E. and Delgado, J.V. The effect of non-genetic factors on the early body weights of Tunisian local goats. Revista Brasileira de Zootecnia. 2010; 39: 1112-1117

4. Ndlovu, L.R. and Simela, L. Effect of season of birth and sex of kid on the production of live weaned single born kids in smallholder East African goat flocks in North East Zimbabwe. Small Ruminant Research.1996; 22:1-6.

5. Najari, S., Gaddour, A. and Ben-hamouda, M. Growth model adjustment of local goat population under pastoral conditions in Tunisian arid zone. Journal of Agronomy.2007; 6:61-67.

6. Dadi, H., Duguma, G., Shelima, B., Fayera, T., Tadesse, M., Woldu, T. and Tucho, T. A. Non-genetic factors influencing post-weaning growth and reproductive performances of Arsi-Bale goats. Livestock Research for Rural Development.2008; 20: 114-119.

7. Zhang, C.; Yang, L. and Shen, Z. Variance components and genetic parameters for weight and size at birth in Boer goat. Livestock Science.2008; 115:72-79.

8. McGregor, B.A. The influence of stocking rate and mixed grazing of Angora goats and Merino sheep on animal and pasture production in southernAustralia. 2. Live weight body condition, carcass yield and mortality. Anim.Prod. Sci. 2010; 50:149-157

9. Gaddour, A., Najari S. and Ouni, M. Kid's growth of pure breeds and crossed caprine genotypes in the coastal oases of southern Tunisia. Research Journal of Agronomy.2007; 2: 51-58.

10. Deribe, B. and Taye, M. Growth performance and carcass characteristics of central highland goats in Sekota District, Ethiopia. Journal of Agricultural Advances.2013; 2:250-258

11. Jimmy, S., David, M., Donald, K. R. and Dennis, M. Variability in body morphometric measurements and their application in predicting live body weight of Mubende and Small East African Goat breeds in Uganda. Middle-East Journal of Scientific Research.2010; 5: 98-105.

12. Chenyambuga, S. W., Komwihangilo, D. M. and Jackson, M. Production performance and desirable traits of Small East African goats in semi-arid areas of Central Tanzania. Livestock Research for Rural Development.2012; 24: 118-124.

13. Macario, J. y Dayenoff, P. Análisis temporal de la percepción del productor caprino malargüino sobre aspectos ligados a la alimentación animal. Primer Congreso Argentino de Producción Caprina. Gobierno de La Rioja. INTA. UMaza. IPAF Cuyo. 
Universidad Nacional de La Pampa. Ley Caprina. La Rioja. 2013; Vol. I: 341-345. ISSN 1853-3647

14. Dayenoff, P.; Jotallan, P.; Duarte, A.; Araya, E.; Banus, G.; Accorinti, C. y Ayala, O. Variación estacional de calidad forrajera de algunos arbustos del sur de Mendoza. IX Congreso Latinoamericano de Especialistas en Pequeños Rumiantes y Camélidos Sudamericanos. Gobierno de La Rioja-ALEPRyCS-INTA-UMaza. La Rioja, Argentina. 2015; Vol I: 281-285. ISSN 2311-0252.

15. Rahman, A. ; Khandoker, M.; Husain,S.; Apu, S.; Mondal A. and Notter, D. Morphometric characterization and relationship of body weight with linear body measurements in Black Bengal buck. Bang. J. Anim. Sci. 2008; 2008. 37:8-16

16. Esteban, C. Razas ganaderas Españolas Caprinas. Madrid: Ministerio de Medio Ambiente, Medio Rural y Marino y Feagas. En Biodiversidad Caprina en Iberoamérica. 2008. 46 pp.

17. Luque, M. Caracterización y evaluación de las razas caprinas autóctonas españolas de orientación cárnica. (Tesis Doctoral). Universidad de Cordoba. Córdoba. España. 2011; 354 pp.

18. Capote, J., Delgado, J. V., Fresno, M., Camacho, E. y Molina, A. Study of morphological variability of the canary goat population. Small Ruminants Research. 1998; 27:167-173

19. Lanari, M. El sistema rural de la cabra criolla neuquina en el norte de la Patagonia. Aspectos socio-económicos culturales de la cría de animales autóctonos Argentina. Series INTA EEA Bariloche. 2004.6pp.

20. Revidatti, M. A., de la Rosa, S. A., Medina, M. A., Sanchez, S., Escalante, F., Cappello-Villada, J. S. y Ribeiro, M. N. Factors influencing birth weight and weight gain after 90 days in goats kids in semiarid region of Formosa (Argentina). $\mathrm{X}^{\circ}$ Congreso de la Asociación Mundial de Caprinos (IGA 2010)

21. Hernandez Zepeda, J. S., Franco Guerrera, F. J., Herrera García, M., Rodero Serrano, E., Sierra Vazquez, A. C., Banuelos Cruz, A. y Delgado Bermejo, J. Estudio de los Recursos Genéticos de México: características morfológicas y morfoestructurales de los caprinos nativos de Puebla. Arch. Zootec.2002; 51:53-64.

22. Chenyambuga, W. and Lekule, F. Breed preference and breeding practices for goats in agro-pastoral communities of semi-arid and sub-humid areas in Tanzania. Livestock Research for Rural Development.2014; Volume 26, Article \#117. 